\title{
THE ENVIRONS OF SUPERNOVA PRECURSORS
}

\author{
O.A. TSIOPA \\ Pulkovo Observatory, St.Petersbourg, 196140 Russia
}

A high activity of a precursor during the last years of its existence can be connected with a close binary system orbit changing.

Some central binary stars in planetary nebulae are thought to be possible supernova precursors. The investigation of the supernova environs formed by the precursor matter before the explosion can help to verify this hypothesis.

Since the SN 1990M event we know that both types of supernovae can exhibit the existence of a rather strong stellar wind ejected prior to the explosion (Polcaro V.F. et al. 1991). The significantly inhomogenous structure of the wind witnesses a high activity of the precursor during the last years of its existence. The wind matter was thrown away as a thin ellipsoidal shell (Tsiopa O.A. 1992). The type Ia supernova precursor being a close white dwarf binary (Iben I.Jr. et al. 1984), the ejected matter can be accelerated by local thermonuclear explosion caused by the surface collision of the components in the periastrum point of the elliptical orbit. Such collisions can take place several times before the orbit becomes round with exentricity decreasing every time. In the case of $1990 \mathrm{M}$ one can estimate the time interval between the collision followed by the wind shell ejection and the supernova explosion of half a year. But it should be indicated that the presence of significant amount of hydrogen so close to the precursor is rather difficult to explain even in terms of a common shell surrounding the system of two white dwarfs.

\section{References}

Iben I.Jr. and Tutukov A.U. (1984). "Supernovae of type I as end products of the evolution of binaries with components of moderate initial mass", Astrophys.J. Supplement, 54, 335-372

Polcaro V.F. and Viotti R. (1991), "H absorbtion in the type-Ia supernova $1990 \mathrm{M}$ during its early phases", Astronomy and Astrophysics, 242, L9-L11

Tsiopa O.A. (1992), "The non-symmetric structure of the stellar wind of the supernova precursor", in "Bipolar outflows and stellar jets". 